\title{
Generalized Fibonacci-Lucas Polynomials
}

\author{
Mamta Singh ${ }^{1}$, Omprakash Sikhwal $^{2 *}$, Vibhoj Parsai ${ }^{3}$ and Yogesh Kumar Gupta ${ }^{4}$ \\ ${ }^{I}$ Department of Mathematical sciences and computer application, Bhundelkhand university, Jhansi (U. P.), INDIA \\ ${ }^{2}$ Department of Mathematics, Mandsaur Institute of Technology, Mandsaur (M. P.), INDIA \\ ${ }^{3}$ Department of Mathematics, P. M. B. Guajarati science college, Indore (M. P.), INDIA \\ ${ }^{4}$ School of studies in Mathematics, Vikram university Ujjain (M. P.), INDIA \\ *Corresponding author E-mail: opbhsikhwal@rediffmail.com
}

Copyright (C) 2014 Omprakash Sikhwal et al. This is an open access article distributed under the Creative Commons Attribution License, which permits unrestricted use, distribution, and reproduction in any medium, provided the original work is properly cited.

\begin{abstract}
Various sequences of polynomials by the names of Fibonacci and Lucas polynomials occur in the literature over a century. The Fibonacci polynomials and Lucas polynomials are famous for possessing wonderful and amazing properties and identities. In this paper, Generalized Fibonacci-Lucas Polynomials are introduced and defined by the recurrence relation $\mathrm{b}_{\mathrm{n}}(x)=\mathrm{xb}_{\mathrm{n}-1}(x)+\mathrm{b}_{\mathrm{n}-2},(\mathrm{x}), \mathrm{n} \geq 2$ with $\mathrm{b}_{0}(x)=2 b$ and $\mathrm{b}_{1}(x)=s$. Some basic identities of Generalized Fibonacci-Lucas Polynomials are obtained by method of generating function.
\end{abstract}

Keywords: Fibonacci polynomials, Lucas polynomials, Generalized Fibonacci polynomials, Generalized Fibonacci-Lucas polynomials.

\section{Introduction}

Various sequences of polynomials by the names of Fibonacci and Lucas polynomials occur in the literature over a century. The Fibonacci polynomials and Lucas polynomials are closely related and widely investigated. Fibonacci polynomials appear in different frameworks. These polynomials are of great importance in the study of many subjects such as algebra, geometry, combinatorics, approximation theory, statistics and number theory itself. Moreover these polynomials have been applied in every branch of mathematics. Fibonacci polynomials are special cases of Chebyshev polynomials and have been studied on a more advanced level by many mathematicians.

Basin, S. L. [1] show that Q matrix generates a set of Fibonacci Polynomials satisfying the recurrence relation $\mathrm{f}_{\mathrm{n}+1}(x)=\mathrm{xf}_{\mathrm{n}}(x)+\mathrm{f}_{\mathrm{n}-1,}(\mathrm{x}), \mathrm{n} \geq 2$ with $\mathrm{f}_{0}(x)=0, \mathrm{f}_{1}(x)=1$.

The Lucas Polynomials are defined by the recurrence formula

$\mathrm{l}_{\mathrm{n}+1}(x)=\mathrm{xl}_{\mathrm{n}}(x)+1_{\mathrm{n}-1},(\mathrm{x}), \mathrm{n} \geq 2$ with $\mathrm{l}_{0}(x)=2, \mathrm{l}_{1}(x)=\mathrm{x}$.

Generating function of Fibonacci polynomials is

$\sum_{n=0}^{\infty} f_{n}(x) t^{n}=t\left(1-x t-t^{2}\right)^{-1}$

Generating function of Lucas polynomials is

$\sum_{n=0}^{\infty} l_{n}(x) t^{n}=(2-x t)\left(1-x t-t^{2}\right)^{-1}$.

Explicit sum formula for (1.1) is given by

$f_{n}(x)=\sum_{k=0}^{\left[\frac{n-1}{2}\right]}\left(\begin{array}{c}n-k-1 \\ k\end{array}\right) x^{n-1-2 k}$,

where $\left(\begin{array}{c}n \\ m\end{array}\right)$ is binomial coefficient and $[\mathrm{x}]$ is the greatest integer less than or equal to $\mathrm{x}$.

Explicit sum formula for (1.10.3) is given by 
$l_{n}(x)=\sum_{k=0}^{[n / 2]} \frac{n}{n-k}\left(\begin{array}{c}n-k \\ k\end{array}\right) x^{n-2 k}$,

where $\left(\begin{array}{c}n \\ m\end{array}\right)$ is binomial coefficient and $[\mathrm{x}]$ is defined as the greatest integer less than or equal to $\mathrm{x}$.

The Fibonacci and Lucas polynomials possess many fascinating properties which have been studied in [2] to [14]. In this paper, Generalized Fibonacci-Lucas polynomials introduced and some basic identities are obtained by method of generating function.

\section{Generalized Fibonacci-Lucas Polynomials}

Generalized Fibonacci-Lucas Polynomials are defined by recurrence relation:

$\mathrm{b}_{\mathrm{n}}(x)=\mathrm{xb}_{\mathrm{n}-1}(x)+\mathrm{b}_{\mathrm{n}-2,}(\mathrm{x}), \mathrm{n} \geq 2$ with initial conditions $\mathrm{b}_{0}(x)=2 \mathrm{~b}$ and $\mathrm{b}_{1}(x)=s$,

where $b$ and $s$ are integers.

The first few generalized Fibonacci-Lucas Polynomials are as follows:

$\mathrm{b}_{0}(x)=2 \mathrm{~b}$,

$\mathrm{b}_{1}(x)=\mathrm{s}$,

$\mathrm{b}_{2}(x)=\mathrm{sx}+2 \mathrm{~b}$.

$\mathrm{b}_{3}(x)=\mathrm{sx}^{2}+2 \mathrm{bx}+\mathrm{s}$ and so on.

If $x=1$, then $b_{n}(1)$ is generalized Fibonacci-Lucas sequence $B_{n}$.

Generating function of Generalized Fibonacci-Lucas Polynomials is

$\sum_{n=0}^{\infty} b_{n}(x) t^{n}=\frac{2 b(1-x t)+s t}{\left(1-x t-t^{2}\right)}$.

Now, we present hyper geometric form of generating function

$$
\begin{aligned}
& \sum_{n=0}^{\infty} b_{n}(x) t^{n}=[2 b(1-x t)+s t]\left(1-x t-t^{2}\right)^{-1}=[2 b(1-x t)+s t][1-(x+t) t]^{-1} \\
& =[2 b(1-x t)+s t] \sum_{n=0}^{\infty}(x+t)^{n} t^{n}=[2 b(1-x t)+s t] \sum_{n=0}^{\infty} t^{n} \sum_{m=0}^{n}\left(\begin{array}{c}
n \\
m
\end{array}\right) x^{n-m} t^{m} \\
& =[2 b(1-x t)+s t] \sum_{n=0}^{\infty} \cdot \sum_{m=0}^{n} \frac{n !}{m !(n-m) !} x^{n-m} t^{m+n} \\
& =[2 b(1-x t)+s t] \sum_{n=0}^{\infty} \cdot \sum_{m=0}^{\infty} \frac{(n+m) !}{m ! n !} x^{n} \cdot t^{2 m+n} \\
& =[2 b(1-x t)+s t] \sum_{n=0}^{\infty} \cdot \frac{(x t)^{n}}{n !} \sum_{m=0}^{\infty} \frac{(n+m) !}{m ! n !} t^{2 m} \\
& =[2 b(1-x t)+s t] e^{x t} \sum_{m=0}^{\infty} \frac{(n+m) !}{n !} \cdot \frac{\left(t^{2}\right)^{m}}{m !} \\
& =[2 b(1-x t)+s t] e^{x t} \sum_{m=0}^{\infty} \frac{\overline{n+m+1}}{\sqrt{n+m}} \cdot \frac{\left(t^{2}\right)^{m}}{m !} \\
& =[2 b(1-x t)+s t] e^{x t} \sum_{m=0}^{\infty}(n+1)_{m} \frac{(1)_{m}}{(1)_{m}} \cdot \frac{\left(t^{2}\right)^{m}}{m !} \\
& =[2 b(1-x t)+s t] e^{x t}{ }_{2} F_{1}\left[n+1: 1,1, t^{2}\right] \text {, } \\
& \sum_{n=0}^{\infty} b_{n}(x) t^{n}=[2 b(1-x t)+s t] e^{x t}{ }_{2} F_{1}\left[n+1: 1,1, t^{2}\right] \text {. }
\end{aligned}
$$

Relation between generalized Fibonacci-Lucas Polynomials, Fibonacci Polynomial and Lucas Polynomials is $b_{n}(x)=s f_{n}(x)+b(2-x t) l_{n}(x)$.

\section{Identities of Generalized Fibonacci Lucas Polynomials}

In this section, some basic identities of Fibonacci-Lucas polynomials have been obtained by method of generating function. 
Theorem 3.1: Prove that

$b_{n+1}(x)-b_{n-1}(x)=x b_{n}(x), \quad n \geq 1$.

Proof: By generating function (2.2), we have

$\sum_{n=0}^{\infty} b_{n}(x) t^{n}=[2 b(1-x t)+s t]\left(1-x t-t^{2}\right)^{-1}$.

Differentiating with respect to $t$, to get

$\sum_{n=0}^{\infty} n b_{n}(x) t^{n-1}=[2 b(1-x t)+s t](x+2 t)\left(1-x t-t^{2}\right)^{-2}+(s-2 b x)\left(1-x t-t^{2}\right)^{-1}$,
$\left(1-x t-t^{2}\right) \sum_{n=0}^{\infty} n b_{n}(x) t^{n-1}=[2 b(1-x t)+s t](x+2 t)\left(1-x t-t^{2}\right)^{-1}+(s-2 b x)$,
$\left(1-x t-t^{2}\right) \sum_{n=0}^{\infty} n b_{n}(x) t^{n-1}=(x+2 t) \sum_{n=0}^{\infty} b_{n}(x) t^{n}+(s-2 b x)$,
$\sum_{n=0}^{\infty} n b_{n}(x) t^{n-1}-\sum_{n=0}^{\infty} x n b_{n}(x) t^{n}-\sum_{n=0}^{\infty} n b_{n}(x) t^{n+1}=\sum_{n=0}^{\infty} n b_{n}(x) t^{n+1}+\sum_{n=0}^{\infty} 2 b_{n}(x) t^{n+1}+(s-2 b x)$.

Equating the coefficient of $\mathrm{t}$, to get

$(n+1) b_{n+1}(x)-x n b_{n}(x)-(n-1) b_{n-1}(x)=x b_{n}(x)+2 b_{n-1}(x)$,

$(n+1) b_{n+1}(x)-(n-1) b_{n-1}(x)=(n+1) x b_{n}(x)$,

$b_{n+1}(x)-b_{n-1}(x)=x b_{n}(x)$.

Theorem 3.2: Prove that

$b_{n}^{\prime}(x)=x b_{n-1}^{\prime}(x)+b_{n-1}(x)+b_{n-2}^{\prime}(x), n \geq 2$.

Proof: By Generating function (2.2), we have

$\sum_{n=0}^{\infty} b_{n}(x) t^{n}=[2 b(1-x t)+s t]\left(1-x t-t^{2}\right)^{-1}$.

Differentiating with respect to $\mathrm{x}$, to get

$\sum_{n=0}^{\infty} b_{n}^{\prime}(x) t^{n}=[2 b(1-x t)+s t](-1)\left(1-x t-t^{2}\right)^{-2}(-t)+\left(1-x t-t^{2}\right)^{-1}(-2 b t)$,

$\left(1-x t-t^{2}\right)^{-1} \sum_{n=0}^{\infty} b_{n}^{\prime}(x) t^{n}=t \sum_{n=0}^{\infty} b_{n}^{\prime}(x) t^{n}-2 b t \sum_{n=0}^{\infty} b_{n}^{\prime}(x) t^{n}-\sum_{n=0}^{\infty} x b_{n}^{\prime}(x) t^{n+1}-\sum_{n=0}^{\infty} b_{n}^{\prime}(x) t^{n+2}=t \sum_{n=0}^{\infty} b_{n}(x) t^{n+1}-2 b t$.

Equating the coefficient of $\mathrm{t}^{\mathrm{n}}$, to get

$b_{n}^{\prime}(x)=x b_{n-1}^{\prime}(x)+b_{n-1}(x)+b_{n-2}^{\prime}(x), n \geq 2$.

Theorem 3.3: Prove that

$b_{n+1}^{\prime}(x)=x b_{n}^{\prime}(x)+b_{n}(x)+b_{n-1}^{\prime}(x), n \geq 1$.

Proof: By 3.1, we have

$b_{n+1}(x)-b_{n-1}(x)=x b_{n}(x), n \geq 1$.

Differentiating with respect to $\mathrm{x}$, to get

$b_{n+1}^{\prime}(x)-b_{n-1}^{\prime}(x)=x b_{n}^{\prime}(x)+b_{n}(x)$,

$b_{n+1}^{\prime}(x)=x b_{n}^{\prime}(x)+b_{n}(x)+b_{n-1}^{\prime}(x)$

Theorem 3.4: Prove that

$n b_{n}(x)=x b_{n}^{\prime}(x)+2 b_{n-1}^{\prime}(x) n \geq 1$ and

$x b_{n+1}^{\prime}(x)=(n+1) b_{n+1}(x)-2 b_{n}^{\prime}(x), n \geq 1$.

Proof: By generating function (2.2), we have

$\sum_{n=0}^{\infty} b_{n}(x) t^{n}=[2 b(1-x t)+s t]\left(1-x t-t^{2}\right)^{-1}$.

Differentiating with respect to $t$, to get 
$\sum_{n=0}^{\infty} n b_{n}(x) t^{n-1}=[s-2 b x]\left(1-x t-t^{2}\right)^{-1}+[2 b(1-x t)+s t]\left(1-x t-t^{2}\right)^{-2}(x+2 t)$.

Differentiating with respect to $\mathrm{x}$, to get

$\sum_{n=0}^{\infty} b_{n}^{\prime}(x) t^{n}=[-2 b t]\left(1-x t-t^{2}\right)^{-1}+[2 b(1-x t)+s t] . t\left(1-x t-t^{2}\right)^{-2}$,

$\sum_{n=0}^{\infty} b_{n}^{\prime}(x) t^{n-1}=[-2 b]\left(1-x t-t^{2}\right)^{-1}+[2 b(1-x t)+s t]\left(1-x t-t^{2}\right)^{-2}$,

$\sum_{n=0}^{\infty} b_{n}^{\prime}(x) t^{n-1}+2 b\left(1-x t-t^{2}\right)^{-1}=[2 b(1-x t)+s t]\left(1-x t-t^{2}\right)^{-2}$.

Using (3.5) in (3.4), to get

$\sum_{n=0}^{\infty} n b_{n}(x) t^{n-1}=[s-2 b x]\left(1-x t-t^{2}\right)^{-1}+(x+2 t)\left\{\sum_{n=0}^{\infty} b_{n}^{\prime}(x) t^{n-1}+2 b\left(1-x t-t^{2}\right)^{-1}\right\}$,

$\sum_{n=0}^{\infty} n b_{n}(x) t^{n-1}=[s-2 b x]\left(1-x t-t^{2}\right)^{-1}+(x+2 t) \sum_{n=0}^{\infty} b_{n}^{\prime}(x) t^{n-1}+2 b(x+2 t)\left(1-x t-t^{2}\right)^{-1}$,

$\sum_{n=0}^{\infty} n b_{n}(x) t^{n-1}=(s-2 b x)\left(1-x t-t^{2}\right)^{-1}+\sum_{n=0}^{\infty} b_{n}^{\prime}(x) t^{n-1}+\sum_{n=0}^{\infty} 2 b_{n}^{\prime}(x) t^{n}+2 b(x+2 t)\left(1-x t-t^{2}\right)^{-1}$.

Equating the coefficient of $\mathrm{t}^{\mathrm{n}-1}$, to get

$n b_{n}(x)=x b_{n}^{\prime}(x)+2 b_{n-1}^{\prime}(x)$.

Equating the coefficient of $\mathrm{t}^{\mathrm{n}}$, to get

$(n+1) b_{n+1}(x)=x b_{n+1}^{\prime}(x)+2 b_{n}^{\prime}(x)$,

$x b_{n+1}^{\prime}(x)=(n+1) b_{n+1}(x)-2 b_{n}^{\prime}(x)$.

Theorem 3.5: Prove that

$(n+1) b_{n}(x)=b_{n+1}^{\prime}(x)+b_{n-1}^{\prime}(x), n \geq 1$.

Proof: By (3.1), we have

$b_{n+1}(x)-b_{n-1}(x)=x b_{n}(x), n \geq 1$.

Differentiating with respect to $\mathrm{x}$, to get

$b_{n+1}^{\prime}(x)-b_{n-1}^{\prime}(x)=x b_{n}^{\prime}(x)+b_{n}(x)$,

$x b_{n}^{\prime}(x)+b_{n}(x)=b_{n+1}^{\prime}(x)-b_{n-1}^{\prime}(x)$.

Using equation (3.5) in equation (3.8), to get

$n b_{n}(x)-2 b_{n-1}^{\prime}(x)+b_{n}(x)=b_{n+1}^{\prime}(x)-b_{n-1}^{\prime}(x)$,

$n b_{n}(x)+b_{n}(x)=b_{n+1}^{\prime}(x)+2 b_{n-1}^{\prime}(x)-b_{n-1}^{\prime}(x)$,

$(n+1) b_{n}(x)=b_{n+1}^{\prime}(x)+b_{n-1}^{\prime}(x)$.

Theorem 3.6: Prove that

$x b_{n}^{\prime}(x)=2 b_{n+1}^{\prime}(x)-(n+2) b_{n}(x), \quad n \geq 0$.

Proof: Using equation (3.5) in equation (3.9), to get

$(n+1) b_{n}(x)=b_{n+1}^{\prime}(x)+\frac{1}{2}\left[n b_{n}(x)-x b_{n}^{\prime}(x)\right]$,

$2(n+1) b_{n}(x)=2 b_{n+1}^{\prime}(x)+\left[n b_{n}(x)-x b_{n}^{\prime}(x)\right]$,

$x b_{n}^{\prime}(x)=2 b_{n+1}^{\prime}(x)+n b_{n}(x)-(2 n+2) b_{n}(x)=2 b_{n+1}^{\prime}(x)+(n-2 n-2) b_{n}(x)$,

$x b_{n}^{\prime}(x)=2 b_{n+1}^{\prime}(x)-(n+2) b_{n}(x)$.

Theorem 3.7: Prove that

$(n+1) x b_{n}^{\prime}(x)=n b_{n+1}^{\prime}(x)-(n+2) b_{n-1}^{\prime}(x), n \geq 1$.

Proof: Using equation (3.3) in equation (3.9), to get

$(n+1)\left\{b_{n+1}^{\prime}(x)-x b_{n}^{\prime}(x)-b_{n-1}^{\prime}(x)\right\}=b_{n+1}^{\prime}(x)+b_{n-1}^{\prime}(x)$, 


$$
\begin{aligned}
& (n+1) b_{n+1}^{\prime}(x)-(n+1) x b_{n}^{\prime}(x)-(n+1) b_{n-1}^{\prime}(x)=b_{n+1}^{\prime}(x)+b_{n-1}^{\prime}(x), \\
& (n+1) b_{n+1}^{\prime}(x)-(n+1) b_{n-1}^{\prime}(x)-b_{n+1}^{\prime}(x)-b_{n-1}^{\prime}(x)=(n+1) x b_{n}^{\prime}(x), \\
& n b_{n+1}^{\prime}(x)-(n+2) b_{n-1}^{\prime}(x)=(n+1) x b_{n}^{\prime}(x), \\
& (n+1) x b_{n}^{\prime}(x)=n b_{n+1}^{\prime}(x)-(n+2) b_{n-1}^{\prime}(x) .
\end{aligned}
$$

Theorem 3.8: (Explicit sum formula) Prove that $b_{n}(x)=2 b \sum_{m=0}^{\left[\frac{n}{2}\right]}\left(\begin{array}{c}n-m \\ m\end{array}\right) x^{n-2 m}$.

Proof: Generating function (2.2), we have

$$
\begin{aligned}
\sum_{n=0}^{\infty} b_{n}(x) t^{n} & =[2 b(1-x t)+s t]\left(1-x t-t^{2}\right)^{-1} \\
& =[2 b(1-x t)+s t]\left(1-x t-t^{2}\right)^{-1} \\
& =[2 b(1-x t)+s t] \sum_{n=0}^{\infty}(x+t)^{n} t^{n} \\
& =[2 b(1-x t)+s t] \sum_{n=0}^{\infty} t^{n} \sum_{m=0}^{n}\left(\begin{array}{c}
n \\
m
\end{array}\right) x^{n-m} \cdot t^{m} \\
& =[2 b(1-x t)+s t] \sum_{n=0}^{\infty} \cdot \sum_{m=0}^{n} \frac{n !}{m !(n-m) !} x^{n-m} t^{m+n} \\
& =[2 b(1-x t)+s t] \sum_{n=0}^{\infty} \cdot \sum_{m=0}^{\infty} \frac{(n+m) !}{m ! n !} x^{n} \cdot t^{2 m+n} \\
& =[2 b(1-x t)+s t] \sum_{n=0}^{\infty} \cdot \sum_{m=0}^{\left[\frac{n}{2}\right]} \frac{(n-m) !}{m ! n !} x^{n-2 m} \cdot t^{n} .
\end{aligned}
$$

Equating the coefficient of $\left(\mathrm{t}^{\mathrm{n})}\right.$ on both sides, to get

$$
b_{n}(x)=2 b \sum_{m=0}^{\left[\frac{n}{2}\right]} \frac{(n-m) !}{m ! n-2 m !} x^{n-2 m} \text {. }
$$

Theorem 3.9: For positive integer $n \geq 0$, prove that $b_{n}(x)=2 b x_{2}^{n} F_{1}\left(\frac{-n}{2}, \frac{-n+1}{2} ;-n, \frac{-4}{x^{2}}\right)$.

Proof: By explicit sum formula (3.12), it follows that

$$
\begin{aligned}
b_{n}(x) & =2 b \sum_{m=0}^{[n / 2]}\left(\begin{array}{c}
n-m \\
m
\end{array}\right) x^{n-2 m} \\
& =2 b x^{n} \sum_{m=0}^{\left[\frac{n}{2}\right]} \frac{(n-m) !}{m ! n-2 m !} x^{-2 m} \\
& =2 b x^{n} \sum_{m=0}^{\left[\frac{n}{2}\right]} \frac{(-1)^{m}(1)_{n}(-n)_{2 m}(-1)^{2 m}(1)_{n} \frac{x^{-2 m}}{m !}}{\left[\frac{n}{2}\right](-1)^{m} 2^{2 m}\left(\frac{-n}{2}\right)_{m}\left(\frac{-n+1}{2}\right)_{m} \frac{x^{-2 m}}{m !}} \\
& \left.=2 b x^{n} \sum_{m=0}^{(-n)_{m}(-1)^{2 m}} \frac{(-n+1}{2}\right)_{m}\left(\frac{-4}{x^{2}}\right)^{m} \\
& =2 b x^{n} \sum_{m=0}^{\left[\frac{n}{2}\right]} \frac{(-1)^{m}\left(\frac{-n}{2}\right)_{m}\left(\frac{-n}{(-n)_{m}} m !\right.}{m !} ; x^{n}{ }_{2} F_{1}\left(\frac{-n}{2}, \frac{-n+1}{2} ;-n, \frac{-4}{x^{2}}\right)^{2}
\end{aligned}
$$


Theorem 3.10: For positive integer $n \geq 0$, prove that

$\sum_{n=0}^{\infty}(c)_{n} b_{n}(x) \frac{t^{n}}{n !}=2 b(1-x t)^{-c} F_{3}\left(\frac{c}{2}, \frac{c+1}{2}, n+1, \frac{n+1}{2}, \frac{n+2}{2}, \frac{t^{2}}{(1-x t)^{2}}\right)$.

Proof: Multiplying both sides of equation (3.12) by $(c)_{n} \frac{t^{n}}{n !}$ and summing between limits $\mathrm{n}=0$ to $n=\infty$, to get

$$
\begin{aligned}
& \sum_{n=0}^{\infty}(c)_{n} b_{n}(x) t^{n}=2 b \sum_{n=0}^{\infty} \sum_{m=0}^{\left[\frac{n}{2}\right]} \frac{n-m !}{m ! n-2 m !}(c)_{n} x^{n=2 m} \frac{t^{n}}{n} \\
& =2 b \sum_{n=0}^{\infty} \sum_{m=0}^{\infty} \frac{n+m !}{m ! n ! n+2 m !}(c)_{n+2 m} x^{n} t^{n+2 m} \\
& =2 b \sum_{n=0}^{\infty} \sum_{m=0}^{\infty} \frac{n+m !}{m ! n !(n+2 m) !}(c+2 m)_{n}(c)_{2 m}(x t)^{n} t^{2 m} \\
& =2 b\left\{\sum_{n=0}^{\infty}(c+2 m)_{n} \frac{(x t)^{n}}{n !}\right\} \sum_{n=0}^{\infty} \frac{n+m !}{m ! n+2 m !}(c)_{2 m} t^{2 m} \\
& =2 b(1-x t)^{-(c+2 m)} \sum_{m=0}^{\infty} \frac{n+m !}{m ! n+2 m !}(c)_{2 m} t^{2 m} \\
& =2 b \sum_{m=0}^{\infty}(1-x t)^{-(c+2 m)} \frac{n+m !}{m ! n+2 m !}(c)_{2 m} t^{2 m} \\
& =2 b(1-x t)^{-c} \sum_{m=0}^{\infty} \frac{n+m !}{m ! n+2 m !}(c)_{2 m}\left[\frac{t^{2}}{(1-x t)}\right]^{m} \\
& =2 b(1-x t)^{-c} \sum_{m=0}^{\infty} \frac{n+m !}{m ! n+2 m !}(2)^{2 m}\left(\frac{c}{2}\right)_{m}\left(\frac{c+1}{2}\right)_{m}\left[\frac{t^{2}}{(1-x t)^{2}}\right]^{m} \\
& =2 b(1-x t)^{-c} \sum_{m=0}^{\infty} \frac{\frac{n+m !}{n !}}{m ! \frac{n+2 m !}{n !}}(2)^{2 m}\left(\frac{c}{2}\right)_{m}\left(\frac{c+1}{2}\right)_{m}\left[\frac{t^{2}}{(1-x t)^{2}}\right]^{m} \\
& =2 b(1-x t)^{-c} \sum_{m=0}^{\infty} \frac{(n+1)_{m}}{m !(n+1)_{2 m}}(2)^{2 m}\left(\frac{c}{2}\right)_{m}\left(\frac{c+1}{2}\right)_{m}\left[\frac{t^{2}}{(1-x t)^{2}}\right]^{m} \\
& =2 b(1-x t)^{-c} \sum_{m=0}^{\infty} \frac{(n+1)_{m}}{2^{2 m} m !\left(\frac{n+1}{2}\right)_{m}\left(\frac{n+2}{2}\right)_{m}}(2)^{2 m}\left(\frac{c}{2}\right)_{m}\left(\frac{c+1}{2}\right)_{m}\left[\frac{t^{2}}{(1-x t)^{2}}\right]^{m} \\
& =2 b(1-x t)^{-c} \sum_{m=0}^{\infty} \frac{\left(\frac{c}{2}\right)_{m}\left(\frac{c+1}{2}\right)_{m}(n+1)_{m}}{m !\left(\frac{n+1}{2}\right)_{m}\left(\frac{n+2}{2}\right)_{m}}\left[\frac{t^{2}}{(1-x t)^{2}}\right]^{m}, \\
& \sum_{n=0}^{\infty}(c)_{n} b_{n}(x) \frac{t^{n}}{n !}=2 b(1-x t)^{-c}{ }_{3} F_{2}\left(\frac{c}{2}, \frac{c+1}{2}, n+1, \frac{n+1}{2}, \frac{n+2}{2}, \frac{t^{2}}{(1-x t)^{2}}\right) .
\end{aligned}
$$

\section{Conclusion}

In this paper, Generalized Fibonacci-Lucas Polynomials have been introduced. Some basic identities are obtained by method of generating function. Also some identities are obtained in hyper geometric form.

\section{Acknowledgements}

The authors would like to thank the anonymous referee for carefully reading the paper and for their comments which greatly improved the paper. 


\section{References}

[1] Basin, S. L., The appearance of Fibonacci Numbers and the Q Matrix in Electrical Network Theory, Mathematics Magazine, Vol. 36, No. 2, (1963), 84-97.

[2] Bicknell, Marjorie. A Primer for the Fibonacci Numbers: part VII - An introduction to Fibonacci Polynomials and their Divisibility Properties, The Fibonacci Quarterly, Vol. 8, No. 4 (1970), 407-420.

[3] Doman, B. G. S. and Williams, J. K., Fibonacci and Lucas Polynomials, Mathematical Proceedings of the Cambridge Philosophical Society 90, Part 3 (1981), 385-387.

[4] Glasson, Alan R., Remainder Formulas, Involving Generalized Fibonacci and Lucas Polynomials, The Fibonacci Quarterly, Vol. 33, No. 3, (1995), 268-172.

[5] Hayes, Richard A., Fibonacci and Lucas polynomials, Master's Thesis, San Jose State college, January, (1965), 36-39.

[6] Hoggatt, V. E. Jr., Private communication of Nov. 17, 1965 to Selmo Tauber, The Fibonacci Quarterly, Vol. 6, (1968), 99.

[7] Hoggatt, V. E. Jr. and Long, C. T., Divisibility Properties of Fibonacci Polynomials, The Fibonacci Quarterly, Vol. 12, No. 2, (1974), 113-120.

[8] Horadam, A. F., Mahon, J. M., Pell and Pell-Lucas Polynomials, Fibonacci Quart., Vol. 23, No. 1 (1985), 7-20.

[9] Koshy, T., Fibonacci and Lucas numbers with Applications, John Wiley and Sons. New York, 2001.

[10] Lupas, A., A Guide of Fibonacci and Lucas Polynomial, Octagon Mathematics Magazine, Vol. 7, No.1 (1999), 2-12.

[11] Singh, B., Bhatnagar,S. and Sikhwal, O., Fibonacci-Like Polynomials and Some Properties, International Journal of Advanced Mathematical Sciences, 1 (3) (2013), 152-157.

[12] Singh, B., Sikhwal, O. and Panwar, Y. K., Generalized Determinantal Identities Involving Lucas Polynomials, Applied Mathematical Sciences, Vol. 3 (2009), No. 8, 377-388.

[13] Swamy, M. N. S., Generalized Fibonacci and Lucas Polynomials and their associated diagonal polynomials, The Fibonacci Quarterly Vol. 37, (1999), 213-222.

[14] Webb, W. A. and Parberry, E. A., Divisibility Properties of Fibonacci Polynomials, The Fibonacci Quarterly Vol. 7, No. 5 (1969), 457-463. 